\title{
Influência de posições do joelho no torque e atividade mioelétrica do tríceps sural na flexão plantar isométrica máxima
}

CDD. 20.ed. 616.7

796.023

http://dx.doi.org/10.1590/1807-55092014000200197

\author{
Marco Túlio BAPTISTA \\ Francisco Xavier Monteiro do NASCIMENTO* \\ Leandro Kegler NARDES* \\ Thiago Torres da MATTA M $/ * * *^{* * *}$ \\ Liliam Fernandes de OLIVEIRA ${ }^{* * * * * *}$
}

\section{Resumo}

Os objetivos do estudo foram comparar o torque isométrico máximo de flexão plantar, em duas posições de joelho e analisar a variação da atividade miolétrica dos músculos do triceps sural nas duas condições. A amostra composta de 14 homens saudáveis. 0 pé direito dos participantes foi fixado ao adaptador do aparelho isocinético com o tornozelo em 90 graus. Duas contrações isométricas voluntárias máximas de cinco segundos foram obtidas para cada posição do joelho: estendido e com 90 graus de flexão. Foram analisados o pico de torque flexor plantar médio e o valor RMS do sinal eletromiográfico (EMG) dos três segundos intermediários do gastrocnêmio lateral (GNL), gastrocnêmio medial (GNM) e sóleo para cada posição. Os torques médios nas posições de joelho em extensão e flexão foram 100,4 \pm 17,4 Nm e 72,6 $\pm 18,4 \mathrm{Nm}$, respectivamente. Os gastrocnêmios apresentaram uma redução do valor RMS relativo com o joelho em flexão $(40,5 \pm 10,5$ e $31,7 \pm 25,6 \%$ para o $\mathrm{GL}$ e GM, respectivamente) enquanto o sóleo aumentou nessa condição $(35,6 \pm 28,9 \%)$. A redução do torque de flexão plantar parece estar associada ao resultado de um compromisso entre a inibição da atividade mioelétrica dos GNM e GNL, e otimização da ativação do sóleo. Os resultados podem contribuir no auxílio da prescrição de exercícios direcionados para o tríceps sural, enfatizando a ativação de diferentes músculos com variações no ângulo articular do joelho.

PalAVRAS-Chave: Contração muscular; Gastrocnêmio lateral; Gastrocnêmio medial; Sóleo; Eletromiografia.

\section{Introdução}

A eletromiografia de superfície é uma ferramenta utilizada para a quantificação da atividade mioelétrica e, quando empregada em combinação com a dinamometria, pode auxiliar na compreensão da participação de cada músculo na produção de um torque articular $^{1-2}$. Em relação ao grupamento muscular do tríceps sural (TS), esta abordagem tem sido bem empregada tendo em vista sua importância funcional nas atividades de vida diária e nos desempenhos esportivos ${ }^{3-5}$, assim como na incidência de traumas acometidos a região do tornozelo ${ }^{6-7}$. Dentre outros fatores, o TS contribui na manutenção da postura ereta, nas várias fases da marcha ${ }^{8}$, na propulsão em movimentos explosivos como o salto e a corrida $a^{4,8-9}$. Além disso, tem importante papel no retorno venoso e, qualquer prejuízo de suas funçôes musculares, pode desencadear uma dificuldade na circulação venosa ${ }^{10}$.

Os músculos sóleo (SOL) e gastrocnêmios (GNs) que compóem o tríceps sural são responsáveis por gerarem a maior parte do torque flexor plantar. Murray et al. ${ }^{11}$ alegam que o TS contribui com, aproximadamente, $80 \%$ do torque, enquanto os cinco músculos perimaleolares contribuem em $20 \%$. Os GNs são biarticulares, agindo como flexores da articulação do joelho e são mais efetivos na flexão plantar do tornozelo com o joelho em extensão.

Durante a flexão da articulação do joelho os músculos GNs perdem eficiência mecânica por se afastarem do comprimento favorável em direção a um encurtamento ${ }^{12-14}$, representado pela parte ascendente da curva torque ângulo ${ }^{1}$. Nesta
*Instituto de Pesquisa da Capacitação Física do Exército.

** Instituto Alberto Luiz Coimbra de Pós-Graduação e Pesquisa em Engenharia, Universidade Federal do Rio de Janeiro.

***Escola de Educação

Física e Desportos, Universidade Federal do Rio de Janeiro. 
condição, a contribuição dos GNs para a produção de torque na flexão plantar seria menor em contração voluntária isométrica máxima (CVIM), resultando em menores valores de torque do que os obtidos com o joelho em extensão. O SOL, por outro lado, não sofre alteração de seu comprimento com a variação do ângulo do joelho e, portanto, sua contribuição para o torque de flexão plantar deve ser similar. Não há consenso na literatura sobre a participação de cada músculo do TS nesta redução de torque $^{12-15}$.

\section{Método}

A amostra foi composta de 14 homens $(18,57 \pm$ $0,64$ anos; $66,9 \pm 7,27 \mathrm{~kg}, 174,5 \pm 9,23 \mathrm{~cm})$ saudáveis, sem antecedentes de lesão do aparelho locomotor e sem experiência prévia do teste a ser realizado. Os indivíduos foram instruídos sobre o protocolo experimental, assinaram o termo de consentimento e orientados a não praticar atividade física no dia do teste. O estudo foi aprovado pelo CEP (031/07).

A instrumentação foi composta por um sistema de aquisição EMG 800C da EMGSystemTM (São
A técnica da eletromiografia de superfície tem sido aplicada para sugerir a contribuição de cada músculo na produção do torque de flexão plantar, baseada na relação EMG-força ${ }^{12,16}$. A relação EMGforça deve ser aplicada com restriçôes, mas é bem aceita para contraçóes isométricas ${ }^{17}$.

Desta forma, o objetivo do estudo foi quantificar a variação do torque isométrico máximo em flexão plantar e analisar a atividade mioelétrica dos três músculos componentes do TS, nas posiçôes de joelho em flexão (JF) e extensão (JE).

Jose dos Campos, Brasil), com rejeição de modo comum de $106 \mathrm{~dB}$ e filtro passa-banda analógico de 10-500 Hz, $2 \mathrm{kHz}$ de taxa de amostragem, conversor de 16 bits A/D. Pares de eletrodos de $\mathrm{Ag}-\mathrm{AgCl}$ com $20 \mathrm{~mm}$ de diâmetro foram posicionados, após a preparação da pele, sobre os músculos gastrocnêmio medial (GNM), gastrocnêmio lateral (GNL) e sóleo (SOL), conforme as recomendaçóes do SENIAM (FIGURA 1) ${ }^{18}$. O eletrodo de referência foi posicionado sobre o maléolo lateral esquerdo.

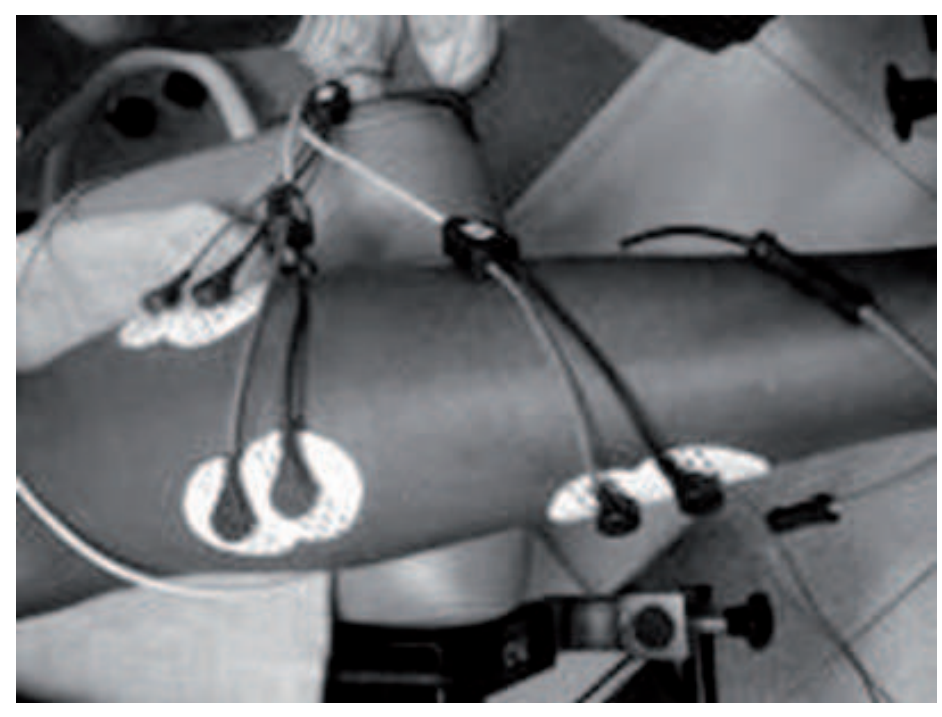

FIGURA 1 - Posicionamento dos eletrodos nos músculos do TS da perna direita, segundo recomendação do SENIAM ${ }^{18}$.

Para medição do torque de flexão plantar foi utilizado um dinamômetro isocinético (Cybex NormTM Medical Systems. Inc. USA). Os sinais de torque e EMG foram sincronizados eletronicamente no sistema de EMG, através de uma entrada auxiliar para leitura dos dados do isocinético exportados via saída analógica.
Os indivíduos foram posicionados em decúbito ventral sobre o dinamômetro com o joelho em extensão $\left(\mathrm{J} 180^{\circ}\right)$ e joelho em flexão a $90^{\circ}\left(\mathrm{J} 90^{\circ}\right)$, estando o tornozelo na posição neutra $\left(90^{\circ}\right)$. Foi desenvolvido um dispositivo para fixação do pé direito ao dinamômetro, para evitar o afastamento do calcanhar durante a contração. 
A sessão de familiarização consistiu de contraçóes submáximas de flexão plantar seguidas por uma tentativa de esforço máximo. Posteriormente, para cada posição do joelho $\left(\mathrm{J} 180^{\circ}\right.$ ou $\mathrm{J}^{\circ} 0^{\circ}$, com ordem foi aleatória), foram obtidas duas contraçóes voluntárias isométricas máximas (CVIM) de cinco segundos com intervalos de 90 segundos. Foi adotado para análise, o teste com o maior valor de torque. Além do incentivo verbal durante o teste, foi utilizado um "feedback" visual da curva de torque.

Antes de cada CVIM, o eixo de rotação do dinamômetro foi cuidadosamente alinhado com o maléolo lateral, ponto central da articulação do tornozelo $^{14}$. O valor RMS, para cada músculo, foi calculado para uma janela de 0,5 segundos, no intervalo de maior torque:

\section{Resultados}

Os resultados médios de torque máximo mostram que o torque com o joelho em flexão de $90^{\circ}$ sofreu uma redução significativa de 28,20 $\pm 12,65 \%$, com valores de $\mathrm{JE}=100,45 \pm 17,45 \mathrm{Nm}$ e $\mathrm{JF}=72,66$ $\pm 18,44 \mathrm{Nm}(\mathrm{p}<0,001)$.

$$
R M S(n)=\sqrt{\frac{\sum_{i=0}^{U-1} e m g(i+n)^{2}}{U}}
$$

Equação 1

A variação relativa $(\% \mathrm{~V})$ do valor RMS, para cada músculo, entre as posiçôes de joelho foi calculada pela razão entre o RMS da posição de JF e JE.

Por não atender ao pressuposto de normalidade em todos os músculos pelo teste de Shapiro-Wilk, foi aplicado o teste não-paramétrico de Kruskal-Wallis para verificar diferenças entre as variaçóes relativas do valor RMS do sinal EMG entre os três músculos. Também foi realizado o "post hoc” de Dunn's caso tenha sido diagnosticada qualquer diferença significativa. O nível de significância adotado foi de $\mathrm{p}<$ 0,05. Os dados foram tratados pelo "software" GraphPad Prism 5.0 (Graphpad Software inc., EUA).

A variação relativa do RMS é apresentada na FIGURA 2, e aponta para uma diferença significativa entre a variação relativa dos gastrocnêmios e a do sóleo $(\mathrm{p}<0,001)$. Não houve diferença significativa entre as variaçóes relativas dos $\mathrm{GN}$ s com a mudança de posição do joelho (FIGURA 2).

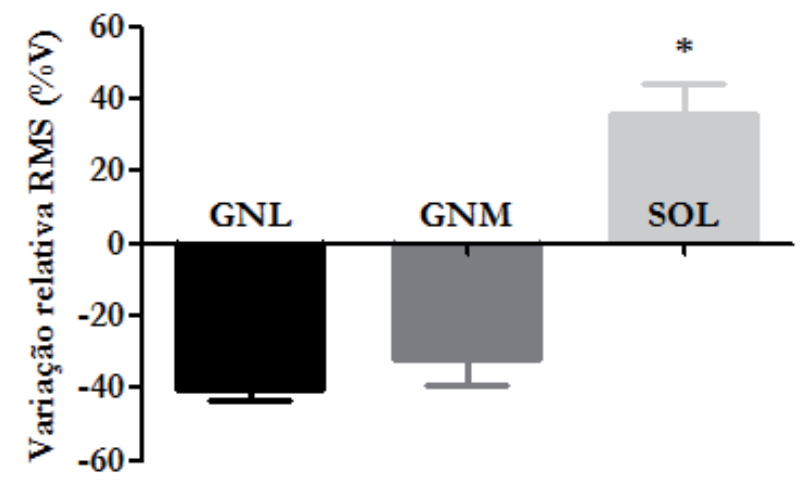
* diferença significativa
entre SOL e os GNs (p
$<0,001)$.

FIGURA 2 - Média e desvio padrão das variações relativas do RMS (\%V) dos três músculos.

\section{Discussão}

Os principais achados do presente estudo estáo relacionados à contribuição mais efetiva do sóleo durante a CVIM com joelho flexionado com acréscimo de aproximadamente $40 \%$, enquanto os GNs possuem comportamento antagônico (redução entre 30 e 40\%). KaWAKAMI et al. ${ }^{19}$ examinou o torque flexor plantar e o valor RMS do TS em seis diferentes combinaçóes angulares entre as articulaçóes do joelho $(\mathrm{J})$ e do tornozelo $(\mathrm{T})$. As combinaçóes $1: 85^{\circ} \mathrm{T} / 160^{\circ} \mathrm{J}$ e $5: 100^{\circ} \mathrm{T} / 100^{\circ} \mathrm{J}$ sáo as que mais se aproximam das posiçốes em extensão e flexão de joelho, respectivamente, adotadas no presente estudo. 
Os autores observaram uma redução de $10,76 \%$ do torque flexor plantar entre essas condições $(101,2 \mathrm{Nm}$ na posição fletida e 113,4 Nm na posição estendida). Na posição fletida, os autores reportaram diminuição significativa do valor RMS do GNM e do GNL quando comparados às outras posiçóes enquanto o SOL não mostrou diferença significativa nos valores de RMS da atividade EMG entre as posiçóes $1 \mathrm{e}$ 5. Nourbakhsh e Kukulka ${ }^{16}$ por outro lado, não encontraram diferenças significativas da atividade mioelétrica dos GNs, porém, o protocolo envolveu uma intensidade de contração de 10 a 30\% CVIM, com o joelho variando de 55 e $90^{\circ}$ de flexão.

A condição uniarticular do sóleo permite supor que sua capacidade de produção de força independe da posição de joelho e, em se tratando de CVIM, era esperada uma ativação mioelétrica máxima deste músculo nas duas posições de joelho. No entanto, foi observado no presente estudo um aumento significativo da ativação mioelétrica do sóleo com o joelho em flexão. Este resultado contradiz aos resultados de Kawakami et al. ${ }^{19}$, mas ratifica Nourbakнsh e KuKulKa $^{16}$ que, mesmo para baixas intensidades, encontraram a ativação relativa superior para sóleo entre as posiçóes de joelho de 55 e $90^{\circ}$ de flexão.

A menor ativação do sóleo com o joelho em extensão, encontrada neste estudo, mesmo em intenção de contração máxima, demonstra que o sistema de controle motor pode apresentar estratégias diferenciadas para o compartilhamento de carga destes músculos. Alguns autores sugerem um mecanismo no qual o sistema nervoso, ao reconhecer a ineficiência mecânica dos GNs para produção de torque de flexão plantar, reduz o nível de ativação destes músculos evitando gasto de energia metabólica ${ }^{20-21}$. O concomitante aumento da atividade do sóleo parece ser dentro deste contexto, uma resposta motora para estabelecer um nível de torque que atenda o compromisso energia/força, como se o sistema nervoso mantivesse uma "reserva" de ativação para músculos que, por impedimento dos agonistas principais, se tornem determinantes na produção do torque articular.

A organização das fibras musculares do sóleo pode explicar como a marcante redução da ativação dos GNs pode ser em parte compensada pela maior participação do sóleo, cujo aumento relativo do valor RMS em torno de $36 \%$ resultou em uma redução do torque de flexão plantar em aproximadamente $28 \%$ com o joelho fletido. Caso a ativação do sóleo invariante em relação à posição de extensão de joelho, esta redução do torque resultaria muito mais acentuada.
O comportamento diferenciado entre as porçôes dos gastrocnêmios é menos estudado. SIRIN e PATLA ${ }^{22}$ examinaram o sinergismo dos flexores plantares durante uma contração sustentada em diferentes condiçôes através de medidas de EMG e torque e demonstraram que o padrão de sinergismo ou compartilhamento de carga foi mais evidente na posição de joelho em extensão do que na posição do joelho em flexão. Os autores postulam que o compartilhamento de carga está relacionado ao nível de intensidade da contração e à eficácia biomecânica dos músculos para a tarefa. $\mathrm{O}$ presente estudo apresentou redução similar da ativação entre as porções do GNs, porém, com um marcante coeficiente de variação (CV) para o comportamento do GNL. O alto valor CV\% (4,78\%) pode ter influenciado nos resultados das comparaçóes entre as duas porçóes dos GNs.

De acordo com KaWAKAMI et al. ${ }^{15}$, a porção medial do GN apresenta uma condição mais desfavorável quando o joelho está flexionado em $90^{\circ}$. Isto se dá pelo menor comprimento da fibra do GNM, que implica em uma menor amplitude efetiva de produção de torque máximo ${ }^{23}$. O GNL, por outro lado, com fibras mais longas, poderia manter níveis suficientes de torque por uma maior variação de comprimento da fibra. $\mathrm{O}$ quanto esta diferença biomecânica pode suscitar uma maior variabilidade de comportamento do GNM em flexão de joelho é incerta e podem estar relacionadas às limitaçóes metodológicas.

Apesar de apresentar uma boa relação EMGforça para contraçóes isométricas, como utilizado neste estudo, o posicionamento dos eletrodos em relação à zona de inervação pode afetar a amplitude do sinal, especialmente quando da mudança da posição do joelho. Além disso, os critérios para SENIAN posicionamento dos eletrodos parece ser imprecisa especialmente em relação ao gastrocnêmio medial $^{18}$. Outro fator importante, é que a ativação dos músculos do tríceps sural humano é sugerida como heterogênea devido a considerável variação na tensão da aponeurose vista em estudos de imagem durante atividade voluntária e confirmado por EMG intramuscular e por EMG multicanal de superfície, sugerindo padróes de ativação diferenciada entre partes distintas de um músculo ${ }^{24-25}$. Em futuros estudos, cuidados metodológicos podem ser testados na tentativa de definir a contribuição de cada músculo na flexão plantar em diferentes posiçóes do joelho.

A redução do torque durante a flexão plantar com o joelho em flexão de $90^{\circ}$, encontrada no presente estudo, é amplamente ratificada na literatura ${ }^{12-15}$. As alteraçóes nos padrões de ativação mioelétrica dos 
músculos do TS são também reportadas, porém com menor consenso. A redução da ativação dos gastrocnêmios é documentada em condiçóes de flexão de joelho ${ }^{19}$, embora outros estudos não tenham confirmado esta redução ${ }^{16,26}$. Porém, os estudos que não corroboram com o presente achado envolvem diferentes protocolos em relação ao nível de ativação solicitado e às posiçóes articulares testadas.

A alteração na posição do joelho afetou significativamente a produção de torque de flexão plantar, com redução de aproximadamente $26 \%$ do torque com joelho em flexão. Nesta posição, a ativação mioelétrica dos gastrocnêmios foi reduzida em torno de $40 \%$ enquanto o sóleo apresentou um aumento de ativação de aproximadamente 39\%. O comportamento do gastrocnêmio lateral foi mais homogêneo entre os indivíduos, enquanto o do gastrocnêmio medial e o do sóleo apresentaram elevados coeficientes de variação.

Estes achados podem contribuir para um programa de treinamento físico ou reabilitação mais direcionada para os componentes do tríceps sural, enfatizando a ativação de diferentes músculos com variaçôes no ângulo articular do joelho.

\begin{abstract}
Influence of knee positions on torque and myoelectric activity of triceps surae at maximum isometric plantar flexion
\end{abstract}

The aim of this study was to compare the maximum torque of plantar flexors using two knee positions and analyze the mioelectric activity variation of the muscles in both conditions. The sample was composed of 14 healthy men. The participants were positioned on the dynamometer with the right foot attached to the adapter in neutral position (ankle at 90 ${ }^{\circ}$. Two five seconds of two consecutives maximum isometric voluntary contractions were obtained for each knee position: full extended and 900. The gastrocnemius lateralis (GNL), medialis (GNM) and soleus RMS value of eletomyography signal and the plantar flexor peak torque average were acquired during three intermediate seconds. The average torques in the knee positions of extension and flexion were $100.4 \pm 17.4 \mathrm{Nm}$ and $72.6 \pm 18.4 \mathrm{Nm}$ respectively. With the knee flexed, the gastrocnemius presented a decreased activation $(40.5 \pm 10.5$ e $31.7 \pm 25.6 \%$ for lateral and medial, respectively) while the soleus showed an increased (35.6 $\pm 28.9 \%)$. The reduction of torque appears to result from a compromise between the inhibition of myoelectric activity of the gastrocnemius medialis and lateralis, while the soleus activation is optimized. The results may contribute to exercise prescription plantar flexors muscles emphasizing the different muscles activation varying the knee position.

KEY WORDS: Muscle contractions; Gastrocnemius lateralis; Gastrocnemius medialis; Soleus; Electromyography.

\title{
Referências
}

1. Lieber RL, Friden J. Functional and clinical significance of skeletal muscle architecture. Muscle Nerve. 2000;23:1647-66.

2. Menegaldo LL, de Oliveira LF. Effect of muscle model parameter scaling for isometric plantar flexion torque prediction. J Biomech. 2009;42:2597-601.

3. Bosco C, Tarkka I, Komi PV. Effect of elastic energy and myoelectrical potentiation of triceps surae during stretchshortening cycle exercise. Int J Sports Med. 1982;3:137-40.

4. Wakeling JM. The recruitment of different compartments within a muscle depends on the mechanics of the movement. Biol Lett. 2009;5:30-4.

5. Pereira GR, de Oliveira LF, Nadal J. Isometric fatigue patterns in time and time-frequency domains of triceps surae muscle in different knee positions. J Electromyogr Kinesiol. 2011;21:572-8.

6. Kinugasa R, Kawakami Y, Fukunaga T. Muscle activation and its distribution within human triceps surae muscles. J Appl Physiol. 2005;99:1149-56.

7. Donken CC, Al-Khateeb H, Verhofstad MH, van Laarhoven CJ. Surgical versus conservative interventions for treating ankle fractures in adults. Cochrane Database Syst Rev. 2012;8:CD008470. 
8. Otis JC, Gage T. Function of the posterior tibial tendon muscle. Foot Ankle Clin. 2001;6:1-14.

9. Goldmann JP, Sanno M, Willwacher S, Heinrich K, Bruggemann GP. The potential of toe flexor muscles to enhance performance. J Sports Sci. 2013;31:424-33.

10. Ishikawa M, Dousset E, Avela J, et al. Changes in the soleus muscle architecture after exhausting stretch-shortening cycle exercise in humans. Eur J Appl Physiol. 2006;97:298-306.

11. Murray MP, Guten GN, Baldwin JM, Gardner GM. A comparison of plantar flexion torque with and without the triceps surae. Acta Orthop Scand. 1976;47:122-4.

12. Kawakami Y, Amemiya K, Kanehisa H, Ikegawa S, Fukunaga T. Fatigue responses of human triceps surae muscles during repetitive maximal isometric contractions. J Appl Physiol. 2000;88:1969-75.

13. Bojsen-Moller J, Hansen P, Aagaard P, Svantesson U, Kjaer M, Magnusson SP. Differential displacement of the human soleus and medial gastrocnemius aponeuroses during isometric plantar flexor contractions in vivo. J Appl Physiol. 2004;97:1908-14.

14. Arampatzis A, Karamanidis K, Stafilidis S, Morey-Klapsing G, DeMonte G, Bruggemann GP. Effect of different ankle- and knee-joint positions on gastrocnemius medialis fascicle length and EMG activity during isometric plantar flexion. J Biomech. 2006;39:1891-902.

15. Kawakami Y, Ichinose Y, Fukunaga T. Architectural and functional features of human triceps surae muscles during contraction. J Appl Physiol. 1998;85:398-404.

16. Nourbakhsh MR, Kukulka CG. Relationship between muscle length and moment arm on EMG activity of human triceps surae muscle. J Electromyogr Kinesiol. 2004;14:263-73.

17. Staudenmann D, Kingma I, Daffertshofer A, Stegeman DF, van Dieen JH. Heterogeneity of muscle activation in relation to force direction: a multi-channel surface electromyography study on the triceps surae muscle. J Electromyogr Kinesiol. 2009;19:882-95.

18. Hermens HJ, Freriks B, Disselhorst-Klug C, Rau G. Development of recommendations for SEMG sensors and sensor placement procedures. J Electromyogr Kinesiol. 2000;10:361-74.

19. Kawakami Y, Abe T, Kanehisa H, Fukunaga T. Human skeletal muscle size and architecture: variability and interdependence. Am J Hum Biol. 2006;18:845-8.

20. Ferguson RA, Ball D, Krustrup P, et al. Muscle oxygen uptake and energy turnover during dynamic exercise at different contraction frequencies in humans. J Physiol. 2001;536(Pt 1):261-71.

21. Arampatzis A, Morey-Klapsing G, Karamanidis K, DeMonte G, Stafilidis S, Bruggemann GP. Differences between measured and resultant joint moments during isometric contractions at the ankle joint. J Biomech. 2005;38:885-92.

22. Sirin AV, Patla AE. Myoelectric changes in the triceps surae muscles under sustained contractions. Evidence for synergism. Eur J Appl Physiol Occup Physiol. 1987;56:238-44.

23. Maganaris CN. Force-length characteristics of the in vivo human gastrocnemius muscle. Clin Anat. 2003;16:215-23.

24. Vieira TM, Loram ID, Muceli S, Merletti R, Farina D. Postural activation of the human medial gastrocnemius muscle: are the muscle units spatially localised? J Physiol. 2011;589(Pt 2):431-43.

25. Staudenmann D, Roeleveld K, Stegeman DF, van Dieen JH. Methodological aspects of SEMG recordings for force estimation--a tutorial and review. J Electromyogr Kinesiol. 2010;20:375-87.

26. Sale D, Quinlan J, Marsh E, McComas AJ, Belanger AY. Influence of joint position on ankle plantarflexion in humans. J Appl Physiol. 1982;52:1636-42.

\section{Agradecimentos}

Os autores gostariam de agradecer à CAPES e FAPERJ como órgãos financiadores de parte deste projeto de pesquisa.

\begin{tabular}{r|l} 
ENDEREÇO & \\
Thiago Torres da Matta & Recebido para publicação: 09/10/2013 \\
R. Conselheiro Lafaiete, 68/904 & 1a. Revisão: 04/02/2013 \\
22081-020 - Rio de Janeiro - RJ - BRASIL & 2a. Revisão: 20/02/2014 \\
e-mail: ttmatta@peb.ufrj.br & Aceito: 20/03/2014
\end{tabular}

202 • Rev Bras Educ Fís Esporte, (São Paulo) 2014 Abr-Jun; 28(2):197-202 